\title{
Optimal Holomorphic Hypercontractivity for CAR Algebras
}

\author{
by \\ Ilona KRÓLAK \\ Presented by Stanisław KWAPIEŃ
}

Summary. We present a new proof of Janson's strong hypercontractivity inequality for the Ornstein-Uhlenbeck semigroup in holomorphic algebras associated with CAR (canonical anticommutation relations) algebras. In the one generator case we calculate optimal bounds for $t$ such that $U_{t}$ is a contraction as a map $L_{2}(\mathcal{H}) \rightarrow L_{p}(\mathcal{H})$ for arbitrary $p \geq 2$. We also prove a logarithmic Sobolev inequality.

1. Introduction. We say that a semigroup $U_{t}$ is hypercontractive if for some $t>0$ it is bounded as a map $U_{t}: L_{r} \rightarrow L_{p}$ for some $\infty>p>r>1$. In 1973 Nelson [N] proved his famous result:

Theorem 1. Let $A_{\gamma}$ be the Dirichlet form operator for the Gauss measure $d \gamma(x)=(2 \pi)^{-n / 2} e^{-|x|^{2} / 2} d x$ on $\mathbb{R}^{n}$. For $1<r \leq p<\infty$ and $f \in$ $L_{p}\left(\mathbb{R}^{n}, \gamma\right)$,

$$
\left\|e^{-t A_{\gamma}} f\right\|_{p} \leq\|f\|_{r} \quad \text { for } t \geq t(r, p)=\frac{1}{2} \ln \frac{p-1}{r-1} .
$$

For $t<t(r, p), e^{-t A_{\gamma}}$ is not bounded from $L_{r}$ to $L_{p}$.

Gross used this inequality to show that the boson energy operator in a model of 2-dimensional Euclidean quantum field theory has a unique ground state. There are several generalizations of that result. The complete version of the hypercontractivity result in its sharp form (optimal time to contraction) for fermions (canonical anticommutation relations) is due to Carlen and Lieb [CL]. Biane [Bia] generalized their result to the case of Bożejko and Speicher's $q$-commutation relations (see [BKSp]) and then in [K2] it was extended to a large class of algebras connected with so called general commutation relations

2010 Mathematics Subject Classification: 81S05, 81R15.

Key words and phrases: hypercontractivity, Fock space, canonical anticommutation relations. 
(see [JSW]). In the non-commutative setting estimates for $p=\infty$ have also been obtained (see $[\mathrm{Bo}]$ ). There is also another sort of extension of Nelson's result. We say that $U_{t}$ is complex hypercontractive if for any holomorphic function $f$ such that $f \in L_{p}\left(\mathbb{R}^{2 n}, \mu\right)$ and for any $p \geq r>0$ there exists $t(r, p)$ such that $\left\|U_{t}(f)\right\|_{p} \leq\|f\|_{r}$ for $t \geq t(r, p)$. In 1983 Janson proved that contractivity in the complex case is attained in a shorter time than in the real case.

Theorem 2. Let $0<r \leq p<\infty$ and let $f \in L_{p}\left(\mathbb{R}^{2 n}, \gamma\right) \cap \operatorname{Hol}\left(\mathbb{C}^{n}\right)$, where $\mathbb{R}^{2 n} \sim \mathbb{C}^{n}$ and $\operatorname{Hol}\left(\mathbb{C}^{n}\right)$ denotes the set of holomorphic functions. Then for $U_{t}$ being a linear extension of $U_{t}\left(H_{n}\right)=e^{-t n} H_{n}$, where $H_{n}$ is the nth Hermite polynomial,

$$
\left\|U_{t}(f)\right\|_{p} \leq\|f\|_{r} \quad \text { for } t \geq t(r, p)=\frac{1}{2} \ln \frac{p}{r} .
$$

For $t<t(r, p), U_{t}$ is not bounded from $L_{r}$ to $L_{p}$.

In $2005 \mathrm{Kemp}[\mathrm{Ke}]$ proved an analog of Janson's complex hypercontractivity inequality for $q$-Gaussian algebras. Precisely, he showed that an analog of the above inequality holds with the same constant for $p$ being any even natural number and $r=2$, i.e. $t(2,2 k)=\frac{1}{2} \ln k$. We propose another proof of his result. We show that the problem of complex hypercontractivity reduces to the one generator case and we calculate explicitly the $L_{p}$ norms of elements of interest in the case of $p$ even using combinatorial methods and for arbitrary $p \geq 1$ using a modified matrix model. We also prove a logarithmic Sobolev inequality.

The paper is organized as follows. In Section 2 we recall the classical representation of the CAR algebra, a construction of an analog of the algebra of holomorphic functions and describe its basic properties. Section 3 contains the first part of the main results. We prove an inequality which reduces the problem to the one generator case and deduce a formula for $L_{2 k}$ norms. In Section 4 we present an alternative matrix model for our algebra, prove the hypercontractive inequality for the one generator case, but without the previous restriction on $p$, i.e. for all $p \geq 2$. As a corollary we get the logarithmic Sobolev inequality.

2. The Clifford algebra. We begin by recalling some well known facts about fermions. The fundamental elements satisfy the canonical anticommutation relations (CAR)

$$
x_{k} x_{j}+x_{j} x_{k}=2 \delta_{k j} \quad \text { for } j, k \in I \text {, a finite index set. }
$$

They can be concretely represented as operators on some Hilbert space. We define the matrices 


$$
\mathrm{Id}=\left(\begin{array}{ll}
1 & 0 \\
0 & 1
\end{array}\right), \quad U=\left(\begin{array}{cc}
1 & 0 \\
0 & -1
\end{array}\right), \quad Q=\left(\begin{array}{ll}
0 & 1 \\
1 & 0
\end{array}\right) .
$$

Let $\mathcal{H}$ denote the $n$-fold tensor product of $\mathbb{C}^{2}$ with itself $(n=|I|)$ :

$$
\mathcal{H}=\underbrace{\mathbb{C}^{2} \otimes \ldots \otimes \mathbb{C}^{2}}_{n \text { times }}
$$

and on $\mathcal{H}$ define

$$
x_{j}=U \otimes \cdots \otimes U \otimes Q \otimes \operatorname{Id} \otimes \cdots \otimes I d,
$$

where $Q$ occurs in the $j$ th place. The operators just defined are easily seen to satisfy the canonical anticommutation relations.

In the non-commutative fermionic holomorphic setting we begin the construction of our algebra $\operatorname{HOL}(I)$ by doubling the number of generators:

$$
\begin{aligned}
x_{k} x_{j}+x_{j} x_{k} & =2 \delta_{k j}, \\
x_{-k} x_{-j}+x_{-j} x_{-k} & =2 \delta_{k j}, \\
x_{-j} x_{i}+x_{i} x_{-j} & =0,
\end{aligned}
$$

where $k=1, \ldots,|I|$. We take

$$
x_{-j}=U \otimes \cdots \otimes U \otimes P \otimes \operatorname{Id} \otimes \cdots \otimes \operatorname{Id} \quad \text { for } P=\left(\begin{array}{cc}
0 & -i \\
i & 0
\end{array}\right),
$$

where $P$ occurs in the $j$ th place.

Denote by $z_{k}$ the operator given by

$$
z_{k}=\frac{1}{2}\left(x_{k}+i x_{-k}\right) \text {. }
$$

Define the holomorphic algebra $\operatorname{HOL}(I)$ as the algebra over $\mathbb{C}$ generated by $\left\{z_{1}, \ldots, z_{|I|}\right\}$, i.e. all polynomials in variables $z_{i}, i \in I$ (the adjoints are not included).

Since $\operatorname{HOL}(I)$ is a subalgebra of $2^{n} \times 2^{n}$ matrices $(n$ being the number of elements in $I$ ), we have on it the standard normalized trace tr. The following lemma contains some properties of the operators $z$ and $z^{*}$. All the statements can be verified by easy calculations (see also [Ke]).

LEMMA 1. The following properties hold:

(3) $\quad\left(z_{j}^{*} z_{j}\right)^{t}=\left(z_{j}^{*} z_{j}\right) \quad$ for $t>0$,

(4) $z_{j}^{*} z_{j} u=u z_{j}^{*} z_{j} \quad$ for $u \in \operatorname{HOL}(I \backslash\{j\})$,

(5) $\quad z_{j} z_{j}^{*}=1-z_{j}^{*} z_{j}$,

(6) $\quad z_{j} z_{j}^{*} z_{j}=z_{j} \quad$ and $\quad z_{j}^{*} z_{j} z_{j}^{*}=z_{j}^{*}$,

(7) $\operatorname{tr}\left(z_{j}^{*} z_{j} u\right)=\operatorname{tr}\left(z_{j}^{*} z_{j}\right) \operatorname{tr}(u)=\frac{1}{2} \operatorname{tr}(u) \quad$ for $u \in \operatorname{HOL}(I \backslash\{j\})$. 
Non-commutative analogs of $L_{p}$ norms can be introduced, namely for $1 \leq p<\infty$ we put

$$
\|A\|_{p}=\left(\operatorname{tr}\left(|A|^{p}\right)\right)^{1 / p}=\left(\operatorname{tr}\left(\left(A^{*} A\right)^{p / 2}\right)\right)^{1 / p} .
$$

We will need an estimate of the $L_{p}$ norm of an arbitrary element $a \in$ $\operatorname{HOL}(I)$. In the next section we will show that the problem of hypercontractivity can be reduced to the one generator case. The proof will go by induction on the number of generators. We end this section with a remark which is the first tool for that reduction.

REMARK 1. Let $a \in \operatorname{HOL}(I)$. Then $a$ can be uniquely decomposed as

$$
a=b+z_{j} c
$$

for any $j \in I$ and $b, c \in \operatorname{HOL}(I \backslash\{j\})$.

For more information about CAR algebras see [BR].

3. Complex hypercontractivity. All the statements of this section remain true also for the so called mixed spin holomorphic algebra $\operatorname{HOL}(I, \sigma)$. Let $\sigma: I \times I \rightarrow\{-1,1\}$ be symmetric, i.e. $\sigma(k, j)=\sigma(j, k)$, and constantly -1 on the diagonal, i.e. $\sigma(k, k)=-1$. Then $\operatorname{HOL}(I, \sigma)$ is the algebra over $\mathbb{C}$ generated by $\left\{z_{1}, \ldots, z_{|I|}\right\}$, where $z_{k}=\frac{1}{2}\left(x_{k}+i x_{-k}\right)$ and

$$
\begin{aligned}
x_{k} x_{j}-\sigma(j, k) x_{j} x_{k} & =2 \delta_{k j}, \\
x_{-k} x_{-j}-\sigma(j, k) x_{-j} x_{-k} & =2 \delta_{k j}, \\
x_{-j} x_{k}-\sigma(j, k) x_{k} x_{-j} & =0 .
\end{aligned}
$$

Lemma 2. Let $b, c \in \operatorname{HOL}(I \backslash\{j\})$, where $j \in I$. Then

$$
\left\|b+z_{j} c\right\|_{2 p} \leq\|\| b\left\|_{2 p}+z_{j}\right\| c\left\|_{2 p}\right\|_{2 p} .
$$

Proof. Without loss of generality we can assume that $j=1$. Denote $z_{1}=z$. We have to prove that

$$
\operatorname{tr}\left(\left[\left(b^{*}+c^{*} z^{*}\right)(b+z c)\right]^{p}\right) \leq \operatorname{tr}\left(\left[\left(\|b\|_{2 p}+\|c\|_{2 p} z^{*}\right)\left(\|b\|_{2 p}+\|c\|_{2 p} z\right)\right]^{p}\right) .
$$

Assign to each sequence from $\{0,1\}^{2 p}$ a term from the left hand side and the corresponding term from the right hand side in the following way:

$$
\left(a_{n}\right) \mapsto \operatorname{tr}\left[\alpha\left(a_{1}\right) \cdots \alpha\left(a_{2 p}\right)\right],
$$

where $\alpha\left(a_{2 k+1}\right)=b^{*}$ if $a_{2 k+1}=0, \alpha\left(a_{2 k+1}\right)=c^{*} z^{*}$ if $a_{2 k+1}=1, \alpha\left(a_{2 k}\right)=b$ if $a_{2 k}=0$ and $\alpha\left(a_{2 k}\right)=z c$ if $a_{2 k}=1$.

Analogously,

$$
\left(a_{n}\right) \mapsto \operatorname{tr}\left[\beta\left(a_{1}\right) \cdots \beta\left(a_{2 p}\right)\right],
$$

where $\beta\left(a_{k}\right)=\|b\|_{2 p}$ if $a_{k}=0, \beta\left(a_{2 k+1}\right)=\|c\|_{2 p} z^{*}$ if $a_{2 k+1}=1$ and $\beta\left(a_{2 k}\right)=$ $z\|c\|_{2 p}$ if $a_{2 k}=1$. 
The corresponding terms are either zero or the term on the left hand side is less than or equal to the term on the right hand side. Assume that there exists a pair of indices $s<j$, both even, such that $a_{s}=1, a_{j}=1$ and for all $s<k<j, a_{k}=0$ (for $l<s$ or $l>j, a_{l}$ can be arbitrary). Then

$$
\operatorname{tr}\left[\alpha\left(a_{1}\right) \cdots \alpha\left(a_{2 p}\right)\right]=\operatorname{tr}\left[\alpha\left(a_{1}\right) \cdots \alpha\left(a_{s-1}\right) z c b^{*} b \cdots b^{*} z c \alpha\left(a_{j+1}\right) \cdots \alpha\left(a_{2 p}\right)\right] .
$$

From Lemma 1 we have

$$
z z_{\underline{j}}=(-1)^{|\underline{j}|} z_{\underline{j}} z, \quad \underline{j}=j_{1} \ldots j_{k}, j_{s} \neq 1, \text { for } s=1, \ldots, k,
$$

where $z_{j}=z_{j_{1}} \cdots z_{j_{k}}$. Also

$$
z z_{\underline{j}}^{*}=(-1)^{|\underline{j}|} z_{\underline{j}}^{*} z, \quad \underline{j}=j_{1} \ldots j_{k}, j_{s} \neq 1, \text { for } s=1, \ldots, k .
$$

Set

$$
\widetilde{d}=\sum_{k=1}^{m} \sum_{|\underline{j}|=k}(-1)^{k} \gamma_{\underline{j}} z_{\underline{j}} \quad \text { for } d=\sum_{k=1}^{m} \sum_{|\underline{j}|=k} \gamma_{\underline{j}} z_{\underline{j}} .
$$

Then since $z^{2}=0$ we get

$$
z c b^{*} b \cdots b^{*} z=z^{2} \widetilde{c} \widetilde{b^{*}} \cdots \widetilde{b^{*}}=0 .
$$

Therefore

$$
\operatorname{tr}\left[\alpha\left(a_{1}\right) \cdots \alpha\left(a_{2 p}\right)\right]=0 .
$$

The same method shows that the corresponding term on the right hand side of the inequality is also zero. In a similar way we find that if there exists a pair of indices $s<j$, both odd, such that $a_{s}=1, a_{j}=1$ and for all $s<k<j, a_{k}=0$, then

$$
\operatorname{tr}\left[\alpha\left(a_{1}\right) \cdots \alpha\left(a_{2 p}\right)\right]=\operatorname{tr}\left[\beta\left(a_{1}\right) \cdots \beta\left(a_{2 p}\right)\right]=0 .
$$

Also if the number of indices $s$ for which $a_{s}=1$ is odd then

$$
\alpha\left(a_{1}\right) \cdots \alpha\left(a_{2 p}\right)=z_{1} d \quad \text { or } \quad \alpha\left(a_{1}\right) \cdots \alpha\left(a_{2 p}\right)=z_{1}^{*} d
$$

for some $d$ which contains neither $z_{1}$ nor $z_{1}^{*}$ and

$$
\beta\left(a_{1}\right) \cdots \beta\left(a_{2 p}\right)=z_{1} w \quad \text { or } \quad \beta\left(a_{1}\right) \cdots \beta\left(a_{2 p}\right)=z_{1}^{*} w, \quad w \in \mathbb{R} .
$$

Therefore in this case

$$
\operatorname{tr}\left[\alpha\left(a_{1}\right) \cdots \alpha\left(a_{2 p}\right)\right]=\operatorname{tr}\left[\beta\left(a_{1}\right) \cdots \beta\left(a_{2 p}\right)\right]=0 .
$$

Take now sequences such that if $a_{s}=1, a_{j}=1$ and $a_{k}=0$ for $s<k<j$ then $s+j$ is odd and the number of indices $s$ for which $a_{s}=1$ is even. Then

$$
\alpha\left(a_{1}\right) \cdots \alpha\left(a_{2 p}\right)=\underbrace{z z^{*} \cdots z z^{*}}_{2 k} d_{1} \cdots d_{2 p},
$$

where $d_{j} \in\left\{b, c, b^{*}, c^{*}, \widetilde{b}, \widetilde{c}, \widetilde{b}^{*}, \widetilde{c}^{*}\right\}$. By Hölder's inequality 


$$
\begin{aligned}
\left|\operatorname{tr}\left(\left(z z^{*}\right)^{k} d_{1} \cdots d_{2 p}\right)\right| & =\frac{1}{2}\left|\operatorname{tr}\left(d_{1} \cdots d_{2 p}\right)\right| \\
& \leq \frac{1}{2}\left\|d_{1}\right\|_{2 p} \cdots\left\|d_{2 p}\right\|_{2 p}=\operatorname{tr}\left(\beta\left(a_{1}\right) \cdots \beta\left(a_{2 p}\right)\right) .
\end{aligned}
$$

The last equality holds because the $L_{2 p}$ norms of any element $d$ and $\widetilde{d}$ in our algebra are the same. Now to end the proof it is enough to apply the triangle inequality.

Now we calculate the norm $\|1+\beta z\|_{2 p}^{2 p}$ as a function of $\beta$. Since it depends only on $|\beta|$, we can assume without any loss of generality that $\beta>0$.

Lemma 3. Take $\beta>0$ and arbitrary $j$. Let $z=z_{j}$ have the properties listed in Lemma 1 . Then for any $p \in \mathbb{N}$ we have $\|1+\beta z\|_{2 p}^{2 p}=\sum_{k=1}^{p} \frac{1}{2} \beta^{2 k} b(p, k)+1, \quad$ where $\quad b(p, k)=\left(\begin{array}{c}p+k \\ 2 k\end{array}\right)+\left(\begin{array}{c}p+k-1 \\ 2 k\end{array}\right)$.

Proof. We have

$$
\begin{aligned}
\|1+\beta z\|_{2 p}^{2 p} & =\operatorname{tr} \underbrace{\left(\left(1+\beta z^{*}\right) \cdots(1+\beta z)\right)}_{2 p \text { terms }} \\
& =\sum_{\underline{\gamma} \in\{0,1\}^{2 p}} \operatorname{tr}\left(\left(1-\gamma_{1}+\gamma_{1} \beta z^{*}\right) \cdots\left(1-\gamma_{2 p}+\gamma_{2 p} \beta z\right)\right) .
\end{aligned}
$$

Since $z_{j}\left(z_{j}^{*} z_{j}\right)^{n}=z_{j}, z_{j}^{*}\left(z_{j} z_{j}^{*}\right)^{k}=z_{j}^{*}$ and $\operatorname{tr}\left(z_{j}\right)=\operatorname{tr}\left(z_{j}^{*}\right)=0$ we can exclude all sequences such that the number of elements in $\left\{j: \gamma_{j}=1\right\}$ is odd. Therefore

$$
\|1+\beta z\|_{2 p}^{2 p}=\sum_{k=0}^{p} \sum_{\substack{\gamma \in\{0,1\}^{2 p} \\ \#\left\{\bar{j}: \gamma_{j}=1\right\}=2 k}} \operatorname{tr}\left(\left(1-\gamma_{1}+\gamma_{1} \beta z^{*}\right) \cdots\left(1-\gamma_{2 p}+\gamma_{2 p} \beta z\right)\right) .
$$

Further, since $z_{j}^{2}=0$, if there exists a pair of indices $j<k$ which are either both odd or both even, $\gamma_{j}=\gamma_{k}=1$ but $\gamma_{s}=0$ for all $j<s<k$ then $\operatorname{tr}\left(\left(1-\gamma_{1}+\gamma_{1} \beta z^{*}\right) \cdots\left(1-\gamma_{2 p}+\gamma_{2 p} \beta z\right)\right)=0$. Denote the set of such $\underline{\gamma}$ by $J$. Now

$$
\begin{aligned}
\|1+\beta z\|_{2 p}^{2 p} & =\sum_{k=0}^{p} \sum_{\substack{\gamma \in\{0,1\}^{2 p} \backslash J \\
\#\left\{j: \gamma_{j}=1\right\}=2 k}} \operatorname{tr}\left(\left(1-\gamma_{1}+\gamma_{1} \beta z^{*}\right) \cdots\left(1-\gamma_{2 p}+\gamma_{2 p} \beta z\right)\right) \\
& =1+\sum_{k=1}^{p} \sum_{\substack{\gamma \in\{0,1\}^{2 p} \backslash J \\
\#\left\{j: \gamma_{j}=1\right\}=2 k}} \operatorname{tr}\left(\left(1-\gamma_{1}+\gamma_{1} \beta z^{*}\right) \cdots\left(1-\gamma_{2 p}+\gamma_{2 p} \beta z\right)\right) \\
& =1+\sum_{k=1}^{p} \sum_{\substack{\gamma \in\{0,1\}^{2 p} \backslash J \\
\#\left\{j: \gamma_{j}=1\right\}=2 k}} \frac{1}{2} \beta^{2 k} .
\end{aligned}
$$


We are left with calculating the number of $\gamma \in\{0,1\}^{2 p}$ such that $\#\{j$ : $\left.\gamma_{j}=1\right\}=2 k$ and $\underline{\gamma} \notin J$ for fixed $k \geq 1$. Take a sequence $j_{1}, \ldots, j_{2 k}$ such that $\gamma\left(j_{1}\right)=\cdots=\bar{\gamma}\left(j_{2 k}\right)=1$. Notice that $j_{s}-j_{s-1}$ must be odd. Then

$$
2 p \geq j_{2 k}=\left(j_{2 k}-j_{2 k-1}\right)+\left(j_{2 k-1}-j_{2 k-2}\right) \cdots+\left(j_{2}-j_{1}\right)+j_{1} .
$$

We have two cases: $j_{1}$ can be either odd or even.

If $j_{1}$ is odd, then the number of such sequences is equal to the number of sequences of $2 k$ odd natural numbers with sum less than or equal to $2 p$. This is equal to the number of sequences of $2 k$ nonnegative even integers with sum less than or equal to $2 p-2 k$. This is the same as the number of sequences of $2 k$ nonnegative numbers with sum less than or equal to $p-k$, and finally it is the same as the number of sequences of $2 k$ positive numbers with sum less than or equal to $p+k$. All these numbers are equal to $\left(\begin{array}{c}p+k \\ 2 k\end{array}\right)$.

Analogously, if $j_{1}-1$ is odd, we are interested in the number of sequences of $2 k$ numbers with sum less than or equal to $2 p-1$. But because we sum up $2 k$ numbers, the sum has to be even, so instead of $2 p-1$ we can write $2 p-2$. Therefore our number is equal to $\left(\begin{array}{c}p+k-1 \\ 2 k\end{array}\right)$. For $p=k$ we set $\left(\begin{array}{c}2 k-1 \\ 2 k\end{array}\right)=0$.

Lemma 4. Let $\beta \in \mathbb{C}$. Take $z$ as in the previous lemma. Then for any natural number $p$,

$$
\|1+t \beta z\|_{2 p} \leq\|1+\beta z\|_{2}
$$

if and only if $|t|^{2} \leq 1 / p$. Additionally, for $\beta \neq 0$ and $p=|t|=1$ the above inequality becomes an equality.

Proof. First notice that $\left\|1+t_{1} \beta z\right\|_{2 p} \leq\left\|1+t_{2} \beta z\right\|_{2 p}$ for $\left|t_{1}\right| \leq\left|t_{2}\right|$. Therefore it is enough to show that

$$
\left\|1+\frac{1}{\sqrt{p}} \beta z\right\|_{2 p}^{2 p} \leq\left(1+\frac{1}{2}|\beta|^{2}\right)^{p}
$$

or equivalently

$$
\|1+\beta z\|_{2 p}^{2 p} \leq\left(1+\frac{1}{2} p|\beta|^{2}\right)^{p} .
$$

Both sides of $(*)$ are polynomials in $|\beta|$. By comparing the corresponding coefficients for $k \geq 2$ we get

$$
\begin{aligned}
& \frac{1}{2}\left(\left(\begin{array}{c}
p+k \\
2 k
\end{array}\right)+\left(\begin{array}{c}
p+k-1 \\
2 k
\end{array}\right)\right) \\
& \quad=\frac{1}{2}\left(\begin{array}{l}
p \\
k
\end{array}\right)\left(\frac{(p+1) \cdots(p+k-1)(p+k)}{(k+1) \cdots(2 k-1) 2 k}+\frac{(p+1) \cdots(p+k-1)(p-k)}{(k+1) \cdots(2 k-1) 2 k}\right) \\
& \quad=\left(\begin{array}{l}
p \\
k
\end{array}\right) \frac{1}{2}\left(\frac{p+1}{k+1}\right)\left(\frac{p+2}{k+2}\right) \cdots\left(\frac{p+k-1}{2 k-1}\right)\left(\frac{p}{k}\right) .
\end{aligned}
$$


Since $\frac{p+s}{k+s}<\frac{p}{s}$ for $s<k \leq p$, we have

$$
\frac{1}{2}\left(\left(\begin{array}{c}
p+k \\
2 k
\end{array}\right)+\left(\begin{array}{c}
p+k-1 \\
2 k
\end{array}\right)\right)<\left(\begin{array}{l}
p \\
k
\end{array}\right) \frac{p^{k}}{2 k !} \leq\left(\begin{array}{l}
p \\
k
\end{array}\right) \frac{p^{k}}{2^{k}} .
$$

For $k=1$ we have $b(p, 1)=p^{2}$. That means that the coefficient of $|\beta|^{2}$ on the left hand side coincides with the corresponding coefficient on the right hand side of $(*)$. This ends the "if" part of the first statement. The "only if" part is covered by Theorem 3 below in a more general setting.

Now we are ready to formulate our theorem (see also [Ke]).

Theorem 3. For any fixed index set $I$ and any $X \in \operatorname{HOL}(I)$ and $U_{t}$ defined as a linear extension of $U_{t}\left(z_{j_{1}} \cdots z_{j_{n}}\right)=e^{-t n} z_{j_{1}} \cdots z_{j_{n}}$ for $j_{1}<$ $\cdots<j_{n}$, we have

$$
\left\|U_{t}(X)\right\|_{2 p} \leq\|X\|_{2} \quad \text { if and only if } e^{-2 t} \leq 1 / p .
$$

This means that the Janson time cannot be improved in the non-commutative setting.

Proof. The sufficiency follows by induction on the number of generators. Fix $j \in I$. Assume that $X \notin \operatorname{HOL}(I \backslash\{j\})$ (i.e. it contains $z_{j}$ ). From Remark $1, X=b+z_{j} c$ for some $b, c \in \operatorname{HOL}(I \backslash\{j\})$ and $U_{t} X=U_{t} b+e^{-t} z_{j} U_{t} c$. From Lemma 2,

$$
\left\|U_{t} X\right\|_{2 p} \leq\|\| U_{t} b\left\|_{2 p}+e^{-t} z_{j}\right\| U_{t} c\left\|_{2 p}\right\|_{2 p} .
$$

Further using Lemma 4 , for $e^{-2 t} \leq 1 / p$ we get

$$
\begin{aligned}
\|\| U_{t} b\left\|_{2 p}+e^{-t} z_{j}\right\| U_{t} c\left\|_{2 p}\right\|_{2 p} & \leq\|\| U_{t} b\left\|_{2 p}+z_{j}\right\| U_{t} c\left\|_{2 p}\right\|_{2} \\
& =\left(\left\|U_{t} b\right\|_{2 p}^{2}+\frac{1}{2}\left\|U_{t} c\right\|_{2 p}^{2}\right)^{1 / 2} .
\end{aligned}
$$

By inductive assumption $\left\|U_{t} b\right\|_{2 p} \leq\|b\|_{2}$ and $\left\|U_{t} c\right\|_{2 p} \leq\|c\|_{2}$ and so

$$
\left\|U_{t} X\right\|_{2 p} \leq\left(\|b\|_{2}^{2}+\frac{1}{2}\|c\|_{2}^{2}\right)^{1 / 2} .
$$

Since $\operatorname{tr}\left(b^{*} z_{j} c\right)=\operatorname{tr}\left(z_{j} c b^{*}\right)=0$ we have

$$
\left\|b+z_{j} c\right\|_{2}^{2}=\left\|z_{j} c\right\|_{2}^{2}+\|b\|_{2}^{2}=\left\|z_{j}\right\|_{2}^{2}\|c\|_{2}^{2}+\|b\|_{2}^{2}=\frac{1}{2}\|c\|_{2}^{2}+\|b\|_{2}^{2} .
$$

Finally

$$
\left\|U_{t} X\right\|_{2 p} \leq\left(\|b\|_{2}^{2}+\frac{1}{2}\|c\|_{2}^{2}\right)^{1 / 2}=\left\|b+z_{j} c\right\|_{2}=\|X\|_{2} .
$$

For the necessity, let $x(\epsilon)=1+\epsilon z$, where $z=z_{j}$ for some $j \in I$. Then

$$
\|x(\epsilon)\|_{2 p}^{2 p}=1+\frac{p^{2}}{2} \epsilon^{2}+o\left(\epsilon^{2}\right)
$$


and

$(\triangle \triangle) \quad\|x(\epsilon)\|_{2}^{2 p}=\left(1+\frac{1}{2} \epsilon^{2}\right)^{p}=1+\frac{1}{2} p \epsilon^{2}+o\left(\epsilon^{2}\right)$.

Hence for $\left\|x\left(e^{-t} \epsilon\right)\right\|_{2 p}^{2 p} \leq\|x(\epsilon)\|_{2}^{2 p}$ we must have

$$
1+\frac{p^{2}}{2} e^{-2 t} \epsilon^{2}+o\left(\epsilon^{2}\right) \leq 1+\frac{1}{2} p \epsilon^{2}+o\left(\epsilon^{2}\right),
$$

and so as $\epsilon \rightarrow 0$, it follows that $e^{-2 t} \leq 1 / p$.

REMARK 2. The equalities $(\triangle)$ and $(\triangle \triangle)$ are also true without the assumption that $p$ is natural. The above necessary condition can be extended to arbitrary $p \geq q \geq 1$, i.e. if $\left\|x\left(e^{-t} \epsilon\right)\right\|_{2 p} \leq\|x(\epsilon)\|_{2 q}$ then $e^{-2 t} \leq q / p$.

4. Matrix model. In this section we present another approach to calculating $L_{p}$ norms of elements of the form $1+\alpha z$. Since in the one generator case, $z$ and $z^{*}$ are $2 \times 2$ matrices, we can calculate the eigenvalues of the selfadjoint, positive operator $|1+\alpha z|^{2}=\left(1+\bar{\alpha} z^{*}\right)(1+\alpha z)$. Indeed,

$$
z=\left(\begin{array}{ll}
0 & 1 \\
0 & 0
\end{array}\right), \quad z^{*}=\left(\begin{array}{ll}
0 & 0 \\
1 & 0
\end{array}\right) .
$$

The $L_{p}$ norm is calculated using the normalized trace on $2 \times 2$ matrices. Then

$$
|1+\alpha z|^{2}=\left(\begin{array}{cc}
1 & \alpha \\
\bar{\alpha} & 1+|\alpha|^{2}
\end{array}\right) .
$$

The characteristic polynomial of that matrix is $W(\lambda)=\lambda^{2}-\left(2+|\alpha|^{2}\right) \lambda+1$, so there are two eigenvalues $\lambda_{1}$ and $\lambda_{2}$ such that $\lambda_{1}+\lambda_{2}=2+|\alpha|^{2}, \lambda_{1} \lambda_{2}=1$ and $\lambda_{1}, \lambda_{2}>0$.

We need the following inequality:

$$
\frac{\lambda_{1}^{p}+\lambda_{2}^{p}}{2} \leq\left(1+p \frac{|\alpha|^{2}}{2}\right)^{p} .
$$

Translating the above to the language of hyperbolic functions by setting $\left(\lambda_{1}+\lambda_{2}\right) / 2=\cosh y$ we get

$$
\cosh p y \leq(p \cosh y+1-p)^{p} \quad \text { for all } y>0, p \geq 1 .
$$

LEMMA 5. For all $\lambda, p \geq 1$,

$$
\frac{\lambda^{p}+(1 / \lambda)^{p}}{2} \leq\left(p\left(\frac{\lambda+1 / \lambda}{2}-1\right)+1\right)^{p} .
$$

Proof. We will prove that

$$
\left(\lambda^{2 p}+1\right) 2^{p-1} \leq\left(p(\lambda-1)^{2}+2 \lambda\right)^{p},
$$


which is equivalent to $(* *)$. Both sides of the above inequality are equal for $\lambda=1$ and arbitrary $p \geq 1$. Define

$$
f(\lambda)=\left(p(\lambda-1)^{2}+2 \lambda\right)^{p}-\left(\lambda^{2 p}+1\right) 2^{p-1} .
$$

Then $f(1)=0$ and

$$
\begin{aligned}
f^{\prime}(\lambda) & =p\left(p(\lambda-1)^{2}+2 \lambda\right)^{p-1}(2 p(\lambda-1)+2)-2^{p} p \lambda^{2 p-1} \\
& =2 p\left[\left(p(\lambda-1)^{2}+2 \lambda\right)^{p-1}(p(\lambda-1)+1)-2^{p-1} \lambda^{2 p-1}\right] \\
& =2 p \lambda^{2 p-1}\left[\left(p\left(1-\frac{1}{\lambda}\right)^{2}+\frac{2}{\lambda}\right)^{p-1}\left(p\left(1-\frac{1}{\lambda}\right)+\frac{1}{\lambda}\right)-2^{p-1}\right] .
\end{aligned}
$$

Notice that $f^{\prime}(1)=0$. Set $x=1-1 / \lambda$. Then

$$
f^{\prime}\left(\frac{1}{1-x}\right)=2^{p} p(1-x)^{-2 p+1}\left[\left(\frac{p}{2} x^{2}+1-x\right)^{p-1}(p x-x+1)-1\right] .
$$

Let $g(x)=\left(\frac{p}{2} x^{2}+1-x\right)^{p-1}(p x-x+1)$. We see that $g(0)=1$. For $p \geq 1, g$ is increasing in $[0,1]$ :

$$
\begin{aligned}
g^{\prime}(x)= & (p-1)\left(\frac{p}{2} x^{2}+1-x\right)^{p-2}(p x-x+1)(p x-1) \\
& +(p-1)\left(\frac{p}{2} x^{2}+1-x\right)^{p-1} \\
= & (p-1)\left(\frac{p}{2} x^{2}+1-x\right)^{p-2}\left(p^{2}-\frac{p}{2}\right) x^{2} \geq 0 \quad \text { for } p \geq 1 \text { and } x \in[0,1] .
\end{aligned}
$$

Thus $g(x) \geq 1$ for $p \geq 1$ and $x \in[0,1]$. This implies that $f^{\prime}(\lambda) \geq 0$ for $\lambda \geq 1$ and $p \geq 1$. We therefore conclude that $f(\lambda) \geq 0$ for $\lambda \geq 1$, which gives the desired result.

Corollary 1. For $y=1+\alpha z$ and $p \geq 2$,

$$
\left\|U_{t}(y)\right\|_{p} \leq\|y\|_{2} \text { if and only if } e^{-2 t} \leq 2 / p .
$$

As a corollary to the optimal complex hypercontractivity inequality we obtain the optimal complex logarithmic Sobolev inequality.

Corollary 2. For $y=1+\alpha z$ and $N(1+\alpha z)=\alpha z$,

$$
\operatorname{tr}\left(|y|^{2} \ln |y|\right)-\|y\|_{2}^{2} \ln \|y\|_{2} \leq \frac{1}{2}\langle N y ; y\rangle .
$$

Proof. From Corollary 1, $\left\|e^{-t N} y\right\|_{2}^{2} \geq\|y\|_{p}^{2}$ for $e^{-2 t}=p / 2$ and $p \geq 2$, i.e. $t \leq 0$. Both sides are continuously differentiable and we will obtain the result by comparing the derivatives at $t=0$. Denote $f(t)=\left\|e^{-t N} y\right\|_{2}^{2}$ and 


$$
\begin{aligned}
& g(t)=\|y\|_{2 e^{-2 t}}^{2} \text {. Then } f^{\prime}(0) \leq g^{\prime}(0) \text {. We calculate } \\
& \quad f^{\prime}(t)=\frac{d}{d t}\left\|e^{-t N} y\right\|_{2}^{2}=\frac{d}{d t}\left\langle e^{-2 t N} y ; y\right\rangle=-2\left\langle N e^{-2 t N} y ; y\right\rangle .
\end{aligned}
$$

For $p=2 e^{-2 t}$,

$$
g^{\prime}(t)=\frac{d}{d t}\|y\|_{2 e^{-2 t}}^{2}=\frac{2\|y\|_{p}}{p}\|y\|_{p}^{1-p}\left(\operatorname{tr}\left(|y|^{p} \ln |y|\right)-\|y\|_{p}^{p} \ln \|y\|_{p}\right)\left(-4 e^{-2 t}\right) .
$$

We take $t=0$ and obtain

$$
\left.g^{\prime}(0)=-4 \operatorname{tr}\left(|y|^{2} \ln |y|\right)-\|y\|_{2}^{2} \ln \|y\|_{2}\right) \quad \text { and } \quad f^{\prime}(0)=-2\langle N y ; y\rangle,
$$

which gives the required inequality.

Acknowledgments. This paper was supported by research grant of Polish Ministry of Science and Higher Education no. N N201 364436 for the years 2009-2012.

\section{References}

[Bia] Ph. Biane, Free hypercontractivity, Comm. Math. Phys. 184 (1997), 457-484.

[Bo] M. Bożejko, Ultracontractivity and strong Sobolev inequality for q-OrnsteinUhlenbeck semigroup $(-1<q<1)$, Infin. Dimens. Anal. Quantum Probab. Relat. Top. 2 (1999), 203-220.

[BKSp] M. Bożejko, B. Kümmerer and R. Speicher, q-Gaussian processes: Non-commutative and classical aspects, Comm. Math. Phys. 185 (1997), 129-154.

[BR] O. Bratteli and D. Robinson, Operator Algebras and Quantum Statistical Mechanics, Springer, 1979.

[CL] E. A. Carlen and E. M. Lieb, Optimal hypercontractivity for Fermi fields and related non-commutative inequalities, Comm. Math. Phys. 155 (1993), 27-46.

[G1] L. Gross, Existence and uniqueness of physical ground states, J. Funct. Anal. 10 (1972), 52-109.

[G2] - Logarithmic Sobolev inequalities, Amer. J. Math. 97 (1975), 1061-1083.

[G3] -, Hypercontractivity and logarithmic Sobolev inequalities for the Clifford-Dirichlet form, Duke Math. J. 42 (1975), 383-396.

[J1] S. Janson, Gaussian Hilbert Spaces, Cambridge Univ. Press, 1997.

[J2] - On hypercontractivity for multipliers on orthogonal polynomials, Ark. Mat. 21 (1983), 97-110.

[J3] -, On complex hypercontractivity, J. Funct. Anal. 151 (1997), 270-280.

[JSW] P. E. T. Jørgensen, L. M. Schmitt and R. F. Werner, Positive representations of general commutation relations allowing Wick ordering, ibid. 134 (1995), 3-99.

[Ke] T. Kemp, Hypercontractivity in non-commutative holomorphic spaces, Comm. Math. Phys. 259 (2005), 615-637.

[KSp] T. Kemp and R. Speicher, Strong Haagerup inequalities for free R-diagonal elements, J. Funct. Anal. 251 (2007), 141-173.

[K1] I. Królak, Wick product for commutation relations connected with Yang-Baxter operators and new constructions of factors, Comm. Math. Phys. 210 (2000), $685-701$. 
[K2] I. Królak, Contractivity properties of Ornstein-Uhlenbeck semigroup for general commutation relations, Math. Z. 250 (2005), 915-937.

[N] E. Nelson, The free Markov field, J. Funct. Anal. 12 (1973), 211-227.

Ilona Królak

Institute of Mathematics

Wrocław University

Pl. Grunwaldzki 2/4

50-384 Wrocław, Poland

E-mail: ikrol@math.uni.wroc.pl

Received January 7, 2010;

received in final form May 3, 2010 